Article

\title{
Distribution of Safety Messages Using Mobility-Aware Multi-Hop Clustering in Vehicular Ad Hoc Network
}

\author{
Rajeshwari Chiluveru $^{1}$ (D), Nishu Gupta ${ }^{2, *(D)}$ and Ariel Soares Teles ${ }^{3}$ (D) \\ 1 Electronics and Communication Engineering Department, Vaagdevi College of Engineering, \\ Warangal 506005, India; chiluverurajeshwari023@gmail.com \\ 2 Electronics and Communication Engineering Department, Chandigarh University, Mohali 140413, India \\ 3 Federal Institute of Maranhão, Sao Luis 65076-091, Maranhão, Brazil; ariel.teles@ifma.edu.br \\ * Correspondence: nishugupta@ieee.org
}

check for updates

Citation: Chiluveru, R.; Gupta, N.; Teles, A.S. Distribution of Safety Messages Using Mobility-Aware Multi-Hop Clustering in Vehicular Ad Hoc Network. Future Internet 2021, 13, 169. https://doi.org/10.3390/fi 13070169

Academic Editor: Paolo Bellavista

Received: 7 June 2021

Accepted: 28 June 2021

Published: 29 June 2021

Publisher's Note: MDPI stays neutral with regard to jurisdictional claims in published maps and institutional affiliations.

Copyright: (c) 2021 by the authors. Licensee MDPI, Basel, Switzerland. This article is an open access article distributed under the terms and conditions of the Creative Commons Attribution (CC BY) license (https:/ / creativecommons.org/licenses/by/ $4.0 /)$.

\begin{abstract}
Reliability and security when distributing safety messages among vehicles in an extremely mobile environment are prominent issues in Vehicular Ad-Hoc Networks (VANETs). In VANET, data transfer becomes challenging because of inherent features such as excessive speed, geographically constrained topologies, unsteady communication links, diversity in the capacity of the channel, etc. A major challenge in the multi-hop framework is maintaining and building a path under such a rigid environment. With VANET, potency in the traffic safety applications has performed well because of the proper design of medium access control (MAC) protocols. In this article, a protocol is proposed pertaining to the distribution of safety messages named mobility-aware multi-hop clustering-based MAC (MAMC-MAC) to accomplish minimum communication overhead, high reliability, and delivery of safety messages in real-time environments. MAMC-MAC has the ability to establish clusteringbased multi-hop sequence using the time-division multiple access (TDMA) technique. The protocol was specially developed for highway outlines to achieve network enhancement and efficient channel usage and guarantees integrity among the vehicles. The performance of the proposed protocol is evaluated using Network Simulator (NS-2), and it demonstrates its superiority over various standard protocols in terms of a number of quality-of-service (QoS)-based parameters. The criteria to select and assess these parameters are their sensitivity and importance to the safety-based applications they provide.
\end{abstract}

Keywords: clustering; contention; multi-hop; TDMA; safety; VANET

\section{Introduction}

Vehicle-to-infrastructure (V2I) and vehicle-to-vehicle (V2V) communication in a Vehicular Ad-Hoc Network (VANET) supports traffic-monitoring, safety, and unsafety applications. When information is transferred through safety applications, it needs high reliability and low delay. Safety application performances are affected when delivering messages, even with negligible delay. Moreover, there is a specific scheduled time period for safety messages to reach the target station. The efficiency of operative safety applications is based on its potential in distributing information immediately by including fairness, high reliability, and flexible network resources usage [1].

Time division multiple access (TDMA)-based clustering scenario gained better recognition for VANET compared with IEEE 802.11 contention-based protocol for improving traffic safety applications due to a large number of increased nodes [2]. From the available traditional protocols (IEEE 802.11), IEEE 802.11p is standardized and wireless access in vehicular environments (WAVE) is used to support low-area communication but is unable to reliably support broadcast services. Broad storms and unrestricted latency cause agony in arbitrary channel access [3]. Accordingly, it experiences large packet loss, access delays, and collisions. All of these issues are randomly correlating with medium access control (MAC)-based contention protocols. Designing Quality-of-Service (QoS)-aware protocols 
is another challenging responsibility in the performance of VANET. This protocol would aim to shorten the delay by assuring the QoS in consideration with the throughput, packet delivery ratio (PDR), and predictable message distribution. In addition, it efficiently uses the network bandwidth [4].

Mostly, VANET routing algorithms use both opportunistic carry-and-forward-based routing techniques and all geography-based routing to master their challenging characteristics. These techniques have authority on either global or local grips over vehicle's state for implementing a multi-hop (hopsector) forwarding scenario to reduce communication overhead while complying with latency constraints from the applications $[5,6]$. With TDMA scheme, many QoS parameters can be improved without any primary domination in order to contribute moderate and predictable data diffusion in V2V communication [7]. Similarly, using a clustering protocol, vehicular schemes can be arranged in a scalable manner. To provide minimum comparable mobility, clustering protocols are used in vehicles to diminish the amount of routing information by splitting the vehicles into groups [8]. In VANET, the clustering method is the best scheme to form fixed clusters with negligible overheads. Perceiving the above issue in VANET, hops (nodes) are partitioned into distinct clusters depending upon their lanes, position, and speed. Moreover, by allocating time periods to distinct nodes, information security is incremented.

The main objective of this work is to develop a scheme that is capable of scaling over a number of hops to convey information in a real-time layout. This requires low latency and high probability of successful delivery (PSD), mainly in the environment where there are less infrastructural facilities for communication. To address these issues while distributing a safety message in multi-hop scenario over highway conditions, we proposed a protocol named distribution of safety message using the MAMC-MAC protocol in VANET. The main characteristics of our proposed work are as follows:

1. To increase the reliability in VANET and delivery safety messages in highway environments for multi-hop scenario, we propose an MAMC-MAC scheme.

2. To maximize the message delivery domain in a real-time scenario, we apply hopsector message directing schema, until four hops;

3. To maximize the throughput and packet delivery ratio of the safety messages, we adopt mobility-dependent clustering of hops;

4. To ensure fairness and reliability in applications, the TDMA scheme is adopted and the available dedicated short-range communication (DSRC) band is divided into frames to achieve maximized channel usage by considering uniformly disseminated vehicular density.

When compared with other schemes, our proposed scheme guarantees channel access to all hops for transmitting safety messages. Another important characteristic of the proposed work is that no cluster head is needed for slot allocation to the hops. We can minimize supplementary overheads and grip in achieving high integrity.

The remaining part of the article is presented as follows. Section 2 describes the related work. In Section 3, the problem explanation is presented. Section 4 determines the proposed system model protocol. Section 5 shows the evaluation and proposed work comparison. Finally, Section 6 concludes the work and defines forthcoming analysis directions.

\section{Related Work}

The key point of cluster-based routing schema is to efficiently categorize the total mobile nodes into sets known as clusters [9]. In [10], a multipath routing algorithm for video transmission was proposed, and the number of challenges experienced in VANET with respect to QoS is minimized. The authors in [11] designed a cluster dependent aggregation-diffusion beaconing process, which is used to maximize connectivity for providing hops within its confined closeness compass of particular locality. In this process, during the aggregation phase, it allows for re-utilization of steady inter-cluster bandwidth. The topology is constructed to diminish the inter-cluster conflicts by generating clusters, which are isolated by the utmost available inter-cluster rift. Therefore, enhancement process 
determines that it has reduced efficiency for inter-cluster dissemination. However, in the intra-cluster dissemination, the possibility of triumphant message acceptance is reduced when the node density is maximized. The above scheme evidently shows that it could not resist under high node density.

There are many existing protocols in VANET to reduce interference using TDMA. For intra-cluster communications in VANET, the authors in [12] developed a mechanism for TDMA slot reservation depending on clustering of vehicles, named time division multiple access cluster-based medium access control (TC-MAC). TDMA slot allocation with rationalized cluster dominating technique is merged with time division multiple access cluster-based medium access control. In this protocol, time periods are allocated to nodes to transfer information without any collision. The task allows vehicles to transmit non-safety messages without affecting the accuracy of receiving and sending, even when there is high traffic density. The authors in [13] proposed an algorithm to increase cluster stability named as dissemination mobility-based clustering algorithm, where stability depends on the time schedule of the cluster heads and on the cluster members (CMs). The above protocols are used in V2V communication for electing cluster heads and for the formation of clusters.

The authors in [14] proposed a motion-prediction time division multiple access-based medium access control scheme that uses a pre-established multi-hop perception scale for distributing MAC slots to share knowledge with adjacent nodes. Nodes select the unused time period depending on the knowledge offered by the surrounding hops to diminish the interferences. The hopsector policy was selected to eliminate the hidden terminal problem (HTP) [15]. Optimal performance can be achieved with two hops, which is displayed in the result. The authors in [16-19] talked in detail about various routing schemes and blockchain framework for securing connected and autonomous vehicles, and critical healthcare services with a broad perspective of the internet of things, using trust-based evaluation system in VANET.

The authors in [20] proposed a multi-hop clustering scheme for VANET. To establish multi-hop clusters, a state-of-the-art mobility mechanism was presented to serve correlative mobility among nodes in multi-hop radius. This scenario presents that hopsector clusters also elongate the communicating area of clusters and attain betterment when differentiated with sole node clusters. The authors in [21] developed a clustering-based medium access control protocol for reducing conflicts in VANET. This scenario employs dynamic multihop clustering to improve network enforcement and provides security applications in highway conditions. The authors in [22] developed a schema for a fifth-generation cellular system to provide wide coverage. The authors in [23] developed an architecture to support data sharing in edge computing. In addition to these, some other works [24,25] inquired about the performance of safety-associated application depending on criteria such as forwarding node ratio, utilization rate of the slot, transmission overhead, end-to-end delay, and throughput.

\section{Problem Explanation}

\subsection{Assumptions and System Model}

We consider multi-hop transmissions in a purely ad-hoc mode scenario, in which a single channel is used to communicate the clustered mobile hops (i.e., nodes). A unique ID is given to each node within a cluster, depending on its MAC address. Each node delivers its packets depending on the routing protocols by operating in the ad-hoc mode. Figure 1 depicts a simple scenario of V2V communication in multi-hop environment. The authors in [26] addressed that, in smart driving applications, a carrier sense multiple access with collision avoidance (CSMA/CA)-dependent medium access control protocol is unable to control the increased message density. Therefore, a likely solution to solve this issue is a TDMA-based MAC. 


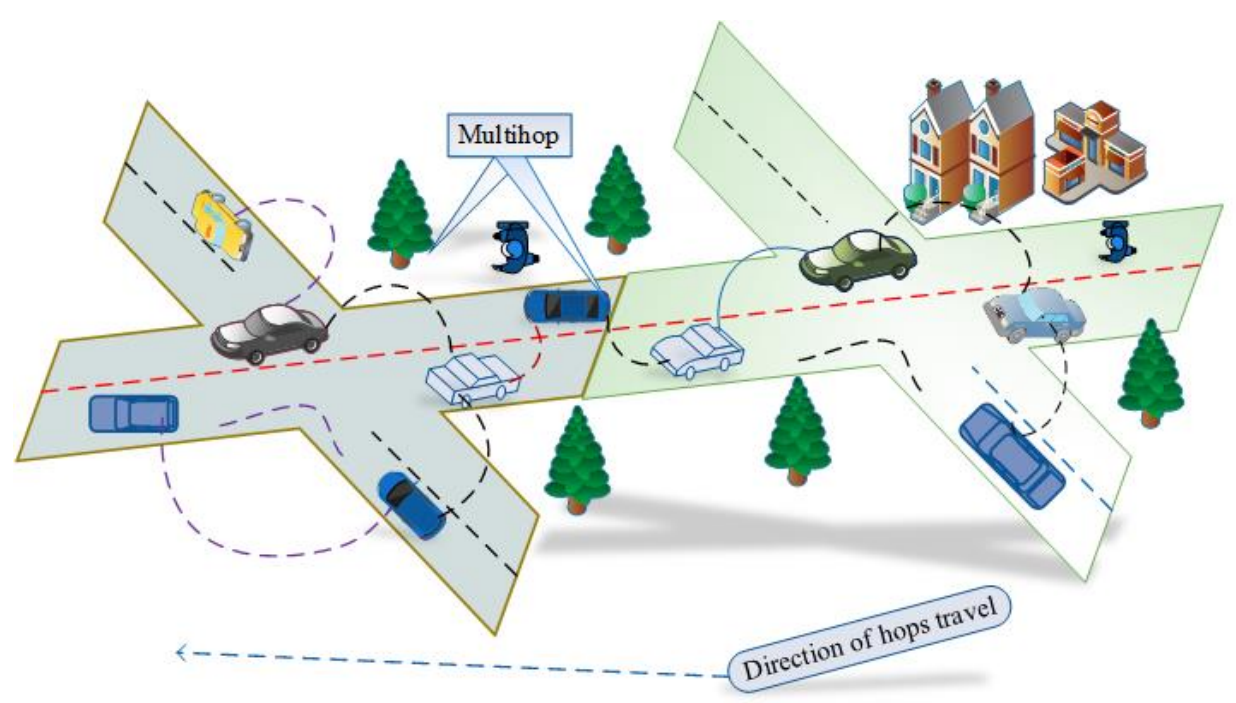

Figure 1. Vehicle-vehicle communication in multi-hop scheme.

In the proposed scenario, we assume a four-lane vehicular environment to provide location-based information to each node by considering an IEEE $802.11 \mathrm{p}$ standard radio device with global positioning system (GPS) installation. Every node contributes its information to their one-hop neighbors about its current position, direction, lane, and speed. By this, we enforce that each safety message produced by a hop inside a cluster should be delivered triumphant to every surrounding clusters in the direction of message travel until the fourth hop $\left(h_{c}\right)$.

\subsection{Objectives}

The aim of the MAMC-MAC protocol is to realize steady diffusion and to transmit event-driven higher-order safety messages by using the available DSRC band. Furthermore, the goal is to reduce the standard delivery latency in the network for decrementing conflicts in a large flow of automobiles, which guarantees the real-time transfer of unstable messages. For realizing our aim, we introduced a scheme based on TDMA for rapid hopsector medium access and a clustering method that accomplishes topology management and alleviates conflicts by always connecting the network. Data dissemination of the present safety message is forwarded over IEEE 802.11 MAC-dependent channels in the assigned period of time.

\section{Execution of MAMC-MAC Protocol}

The proposed MAMC-MAC is divided into three stages. During the primary stage, cluster formation occurs; here, all of the nodes are grouped as distinct clusters on the basis of their speed. During the second stage, TDMA time slot allocation occurs. The main motivation behind TDMA schema employment is to guarantee successful transfer of safety messages. In VANET, the safety applications always aim to achieve less delay, successful transmission, and time slots allocated to every node by transferring safety messages without interrupting other nodes, which are challenging. TDMA stands out best in assigning different time periods to each cluster inside the network. During the third stage, progressive hopsector safety message distribution takes place. Progressive hopsector is defined as an aggressive message routing schema in which messages progress ahead to hops that are arranged superior to handover them to upcoming hops. In the vehicular scenario, the purpose of multi-hop routing is to elevate the broadcasted messages transmission range. However, for the effective multi-hop progressing, heavy traffic scenarios are considered since there are better positioned nodes enclosed by the communication range [27]. In the next subsections, the proposed algorithm phases are explained. The layout of the MAMCMAC protocol is shown in Algorithm 1. 


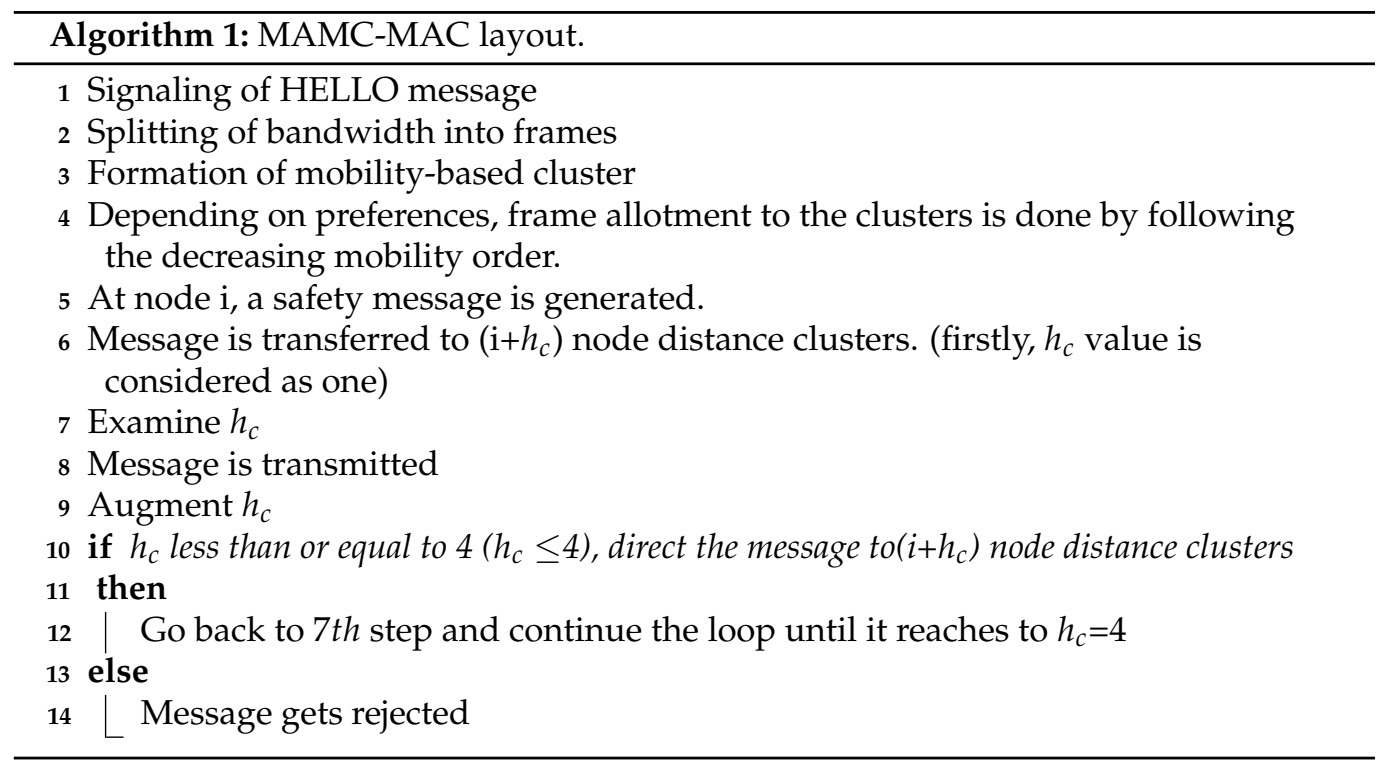

\subsection{Clustering Mechanism}

Our proposed scenario makes use of the clustering topology for safety message distribution. Clustering of nodes is performed depending on its mobility. A cluster is formed by combining nodes with the same average speed. The multi-hop topology and single-hop topology are used for linking various clusters and the nodes within a cluster. The clustering algorithm requires only the distribution of status messages of the node's, since it is a mobility-based algorithm. Therefore, for the first time, whenever these nodes are employed on the roadside, they begin to convey their status information without an elected cluster heads ( $\mathrm{CHs})$. Once all of the nodes in the network receive these messages by range, they start forming a cluster, admiring each other's mobility patterns. Maximum average speed clusters is given preference for safety message distribution, which can be accomplished by the TDMA mechanism. The mechanism for this is shown in Algorithm 2.

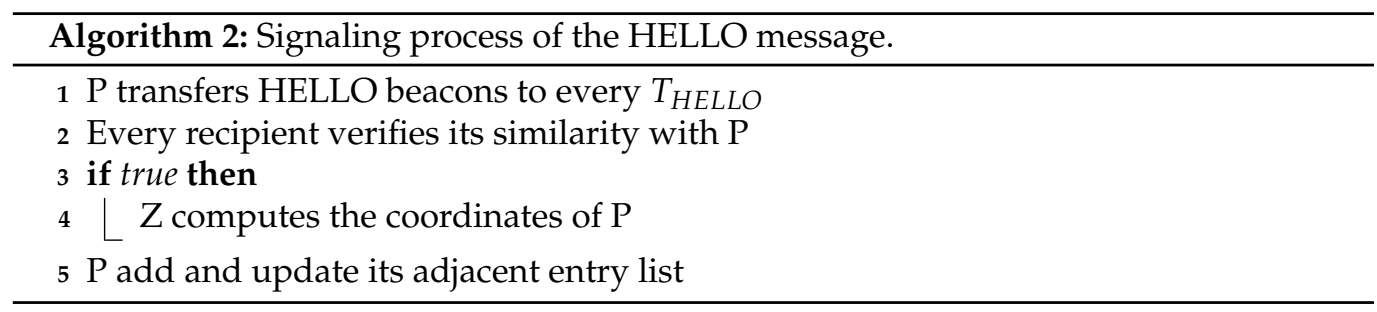

All of the nodes within the system broadcasts HELLO messages to maintain positioning information. The HELLO message contains information about mobility range, position, and node ID. The HELLO message transmission period is denoted by $T_{H E L L O}$. In case a HELLO message is received by some node $Z$, it verifies its likeness with another node P. A node accepts the surrounding nodes travelling in a similar direction by restricting the transfer of the traffic in the opposite direction. A neighboring cluster's positional information is recorded by broadcasting the HELLO messages. These data are given as the input to the clustering algorithm.

Cluster-dependent routing protocol has four phases: selecting the cluster head, data aggregation, formation of cluster, and transmission of data. Figure 2 shows mobilitydependent clustered hops. This scheme produces comparatively steady clustering framework by invalidating the cluster head election overhead. Short duration cluster is given superior access to the channel. Later, channel access is given to a long duration cluster. Anyhow, by including the variation of speed, the partitioning of the network is performed with 
a minimum number of clusters in such a way that the node's distribution with the highest possibility is conducted depending on its mobility structure. Our developed scheme usesthe TDMA schema for allocating time periods to clusters for intra-cluster communication. This is performed by determining the hop for transmitting the message, depending on the slots' availability in the cluster. Cluster formation is performed with similar direction nodes. Accordingly, in our work, we considered all of the surrounding hops that travels in the similar direction. Though the variation in speed level is high due to this, surrounding hops cannot be included in the cluster.

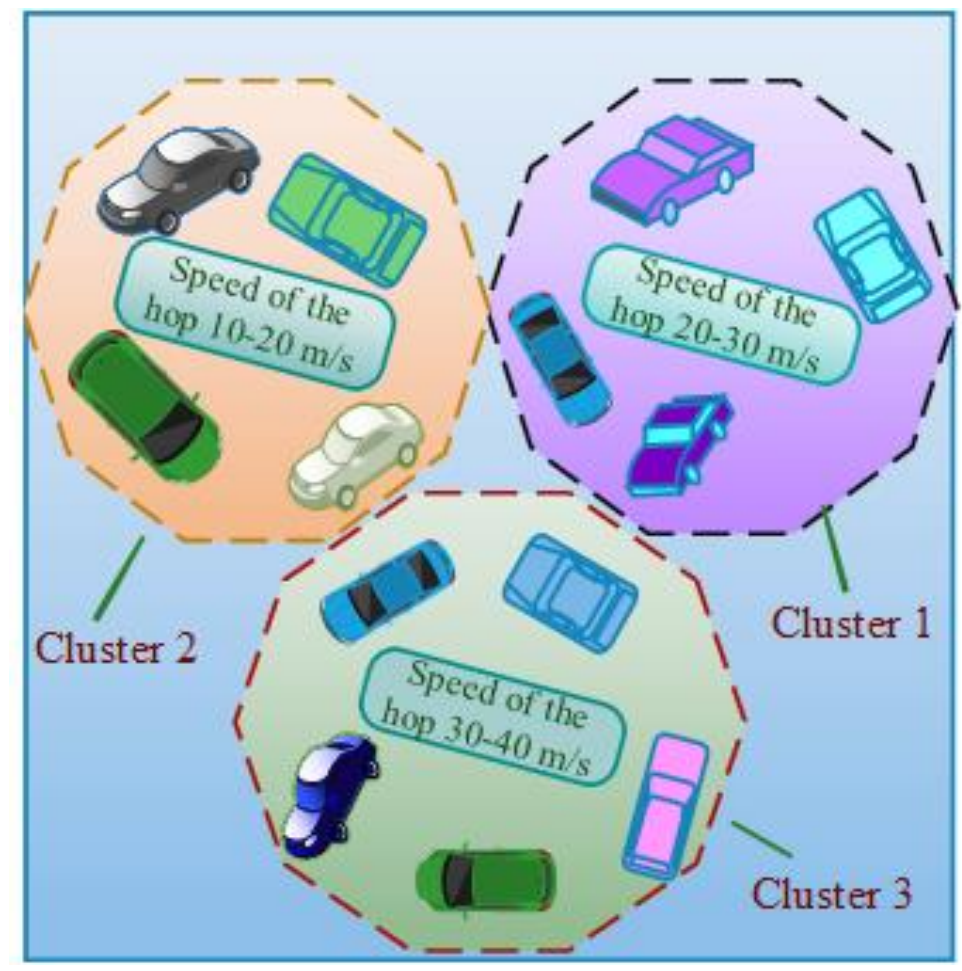

Figure 2. Mobility-dependent clustering scheme.

Our developed protocol achieves minimum interference and incremented bandwidth usage when the nodes are consistently dispersed on the roads. However, the slot requirement will vary when vehicular density is nonuniform, thus leading to interference, inefficient bandwidth utilization, and broadcast storming. Due to this reason, we considered the constant vehicular density and the hops travelling with constant speed during the simulation time. The assumed realistic slot of $2.5 \mathrm{~min}$ coins justify our work. The formation of such expectations aims to overcome the overhead carried by $\mathrm{CH}$ election process.

\subsection{Allocation of TDMA Time Period}

The reason behind using the TDMA schema is to allocate time frames in one-hop interdistance radius to every cluster by supporting contention less channel access. Anyhow, until the arrival of transmission time, the cluster members have to wait when there are a large number of nodes at the destination receiving messages. We remove this latency by preferring time allocation to clusters in decreasing order depending on its mobility. The procedure for frame allotment must satisfy the condition that it is allowed to process the packet to only one neighboring hop, in the reverse direction of the node movement.

As discussed in the IEEE 1609.4 WAVE standard, the developed schema rules out the implementation of channel switching during the synchronization interval. Irrespective of service channel interval (SCHI) and control channel interval (CCHI), the message is forwarded to all other channels. Here, the complete DSRC bandwidth $(75 \mathrm{MHz})$ is divided into slots. Every cluster is assigned a frame for enabling multi-hop broadcast with minimal delay. Each frame is further categorized into slots. Frame assignment is performed based 
on cluster mobility, in which priority is given to the highest mobility cluster. The accessing time to the channel is less for clusters with maximum speed. Figure 3 describes the frame assignment procedure based on clustering.

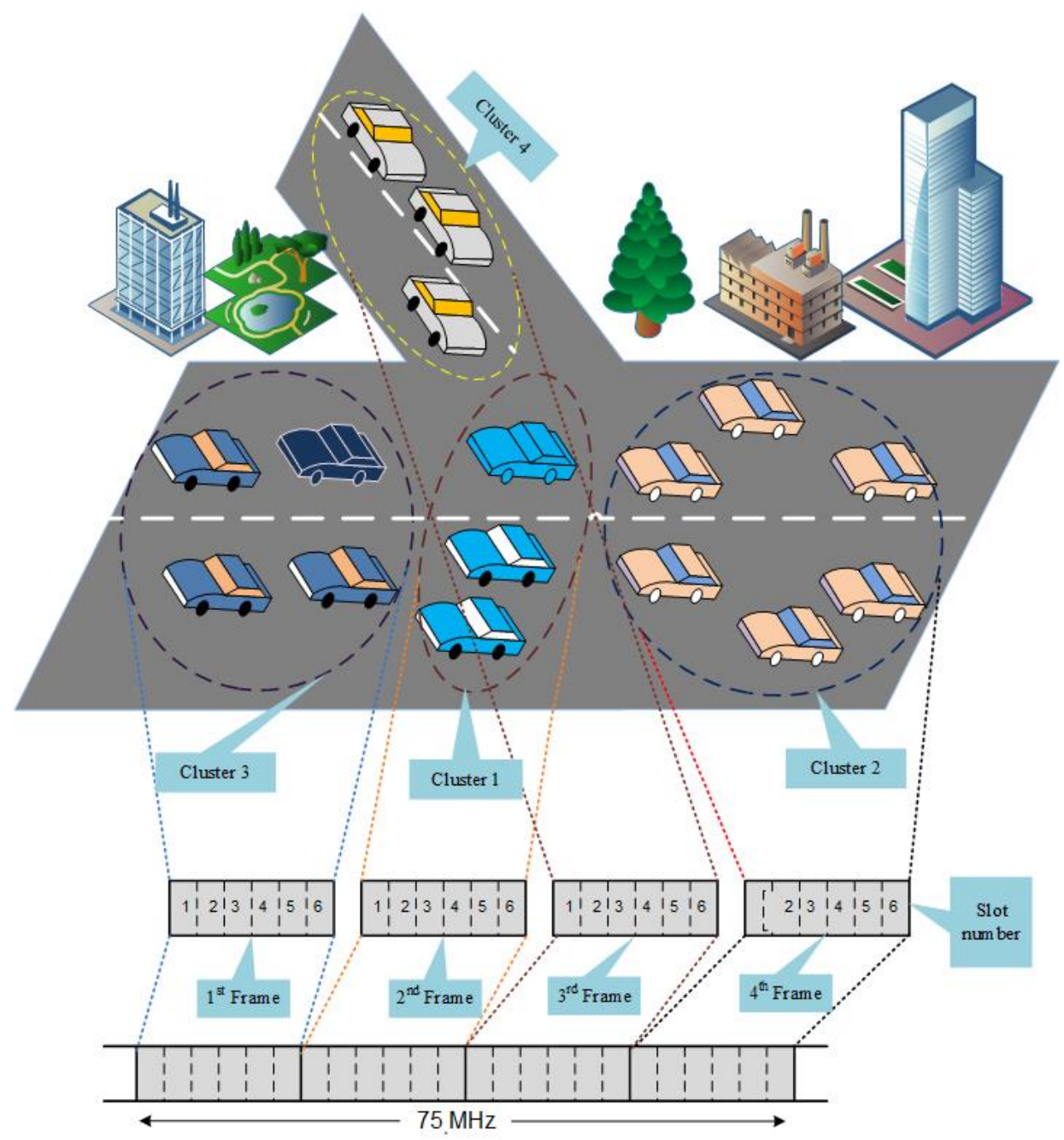

Figure 3. Allocation of the TDMA time period depending on clustering.

In the MAMC-MAC protocol, for transmission, at the start of each TDMA time slot, the hop arbitrarily chooses a transmit time period in which each slot has the equal probability to be chosen by a node. To make it comparable to WAVE's control channel (CCH), every TDMA frame has 20 slots, with a time duration of $1 \mathrm{~ms}$ for immediate broadcast of the safety messages. The hops distribute the messages. Therefore, a slot becomes vacant within the assigned frame to allow the upcoming messages from neighboring hops. From this, the frame distribution is performed among the nodes within the network, since all nodes are familiar with the information on the unallocated slots. The clustering algorithm describes the number of frames per cluster that is fed as a MAC layer input. The slot assignment is performed in a manner that; when a hop collects a message moving in a specific direction, it processes it right away to its next hop in the similar direction [21]. For inter- and intra-cluster communication, the proposed MAMC-MAC designed a scheme by dividing the time periods of TDMA frame according to our requirements.

From Figure 4, we can observe that a TDMA frame is divided into ' $q$ ' time periods beginning with the $0 t h$ slot and ending at the ' $q-1 t h$ ' slot. Synchronization of the first TDMA frame is performed by the 0 th slot. Later, it transfers the slot-assignment state (SAS) enclosed in the specified cluster such that each hop is provided with dedicated time slot 
for data transmission. In the Figure 4 , the 1 st slot to $q-1$ th slots are used for transmission of data.

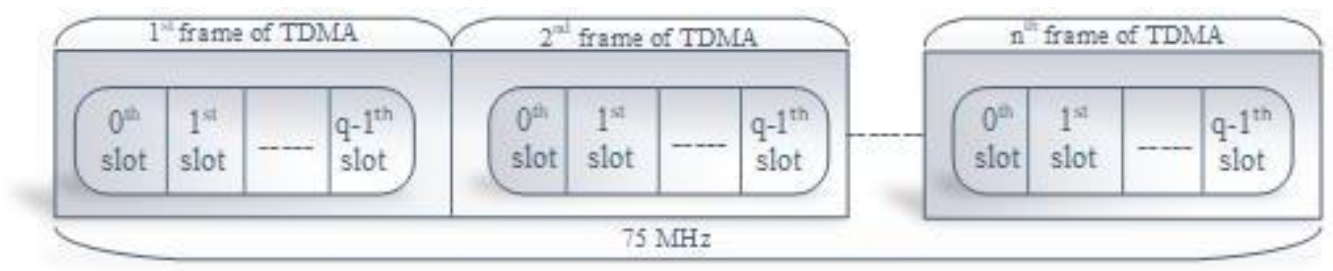

Figure 4. TDMA frame format.

\subsection{Multi-Hop Message Routing}

The major challenge in multi-hop message delivery is choosing the best next node path to route the data. The next node is selected based on the hops present in the direction of the destination hop. This maximizes the possibility of detecting the shortest path [28]. We consider P hops in the network, say the location of a random cluster a1 is $P_{a 1}$. It is clear from the below equation (Equation (1)) that, during any time slot ' $t$ ', if any two clusters are enclosed in RF range of each other, they satisfy the following equation:

$$
P=\frac{\frac{A_{\mathrm{n} 1}(t)}{\left(P_{\mathrm{a} 1}(t)-P_{\mathrm{a} 2}(t)\right)^{2}}}{\frac{\sum P_{\mathrm{a} 1}(t)}{\left(P_{\mathrm{a} 1}(t)-P_{\mathrm{a} 2}(t)\right)^{2}}}>S I N R
$$

where $A_{n 1}(t)$ gives the node $\mathrm{n} 1$ transmission power, $P_{a 1}(t)-P_{a 2}(t)$ gives the inter cluster distance of two clusters, SINR gives the signal-to-interference-and noise ratio, while $\sum A_{a 1}(t)$ gives the cluster a1 summarized transmission power. Equation (2) states that the two clusters (a1 and a2) are coupled at some random time ' $t$ ' if transmission range $T_{r}$ is greater than the distance between them.

$$
\left(P_{\mathrm{a} 1}(t)-P_{\mathrm{a} 2}(t)\right)<T_{\mathrm{r}}
$$

In multi-hop scenario, the message broadcasting is performed very fast amid the nodes connected with a message routing schema. Here, a hop within a cluster transfers data packets to its surrounding clusters and every cluster after successful reception of the packet forwards it to the next neighboring cluster. To ensure transmission efficiency of messages, it is necessary to have an effective routing method, as shown in [29]. The multi-hop message routing algorithm is shown in Algorithm 3.

The flowchart of the proposed MAMC-MAC protocol is shown in Figure 5. First, HELLO message signaling grants nodes within the network to become familiar with each other's coordinates. Next, in our approach, we split the complete DSRC band into frames so that the complete bandwidth is available for transmission of a safety message. Later, clusters are formed depending upon the mobility pattern congregated to beaconing of the HELLO message. Furthermore, frame assignment to the clusters is performed depending upon their mobility pattern. This implies that a greater number of frames are assigned to the clusters with maximum speed. This guarantees the successful transmission and strengthens channel utilization. These frames are further split into a number of slots. For transmitting a message, each node is assigned to a slot. Now, consider initiation of the safety message at node $\mathrm{i}$ and transferred to the clusters with a hop distance of $\left(\mathrm{i}+h_{c}\right)$, with $h_{c}$ representing the message hop count. At first, we consider the $h_{c}$ value to be 1 . Whenever the clusters near one hop receives the message, it is verified for the present $h_{c}$. If the $h_{c}$ value is less than $M$, for $M=4$, the message is transmitted and the value of $h_{c}$ is augmented by 1 . Similarly, the message is relayed and broadcasts up to M nodes far away clusters. We assume $h_{c}$ to be 4 because the message is no longer relayed when $h_{c}$ becomes greater than $\mathrm{M}$ hops and is discarded as obtained from the performance evaluation. 

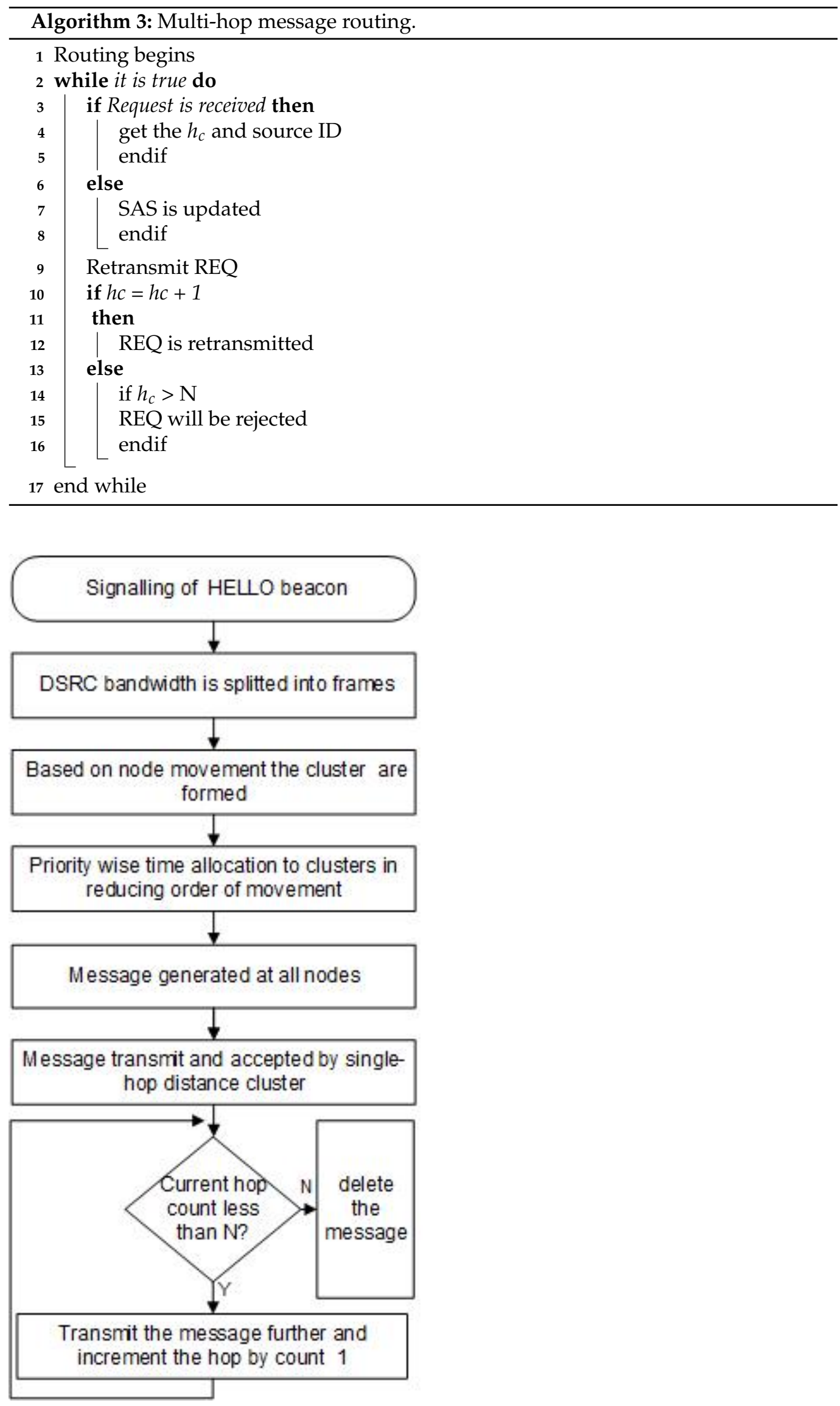

Figure 5. MAMC-MAC flowchart. 


\section{Results and Discussion}

The performance of the proposed protocol is evaluated and compared with (i) existing protocols such as WAVE-enhanced service message delivery (WSD), (ii) the IEEE 802.11p standard [30], (iii) a distributed multi-channel mobility-aware cluster that depends on medium access control (DMMAC) [31], and (iv) a cluster-based beacon dissemination process (CB-BDP) [32]. The objective of this experimental evaluation was to improve the safety at the road level by considering QOS requirements of vehicular applications. An efficient $\mathrm{MAC}$ protocol was developed to reduce the impact of relative speed in $\mathrm{V} 2 \mathrm{~V}$ communication by providing preferences to the hop before it leaves the range of communication. Simulation is performed on a Network Simulator (NS-2) for up to four relay nodes in the hopsector scenario.

\subsection{Parametric Setup}

Performance is evaluated on a four-bypass highway environment with bidirectional hops movement. The speed of the node varies between 10 and $40 \mathrm{~m} / \mathrm{s}$. In a multi-hop ad-hoc region, every node in V2V communication uses similar IEEE 802.11p standard MAC specifications. The node transmission area is up to $0.3 \mathrm{~km}$, and the time taken for simulation is $2.5 \mathrm{~min}$, with a data rates of up to $6000 \mathrm{kbps}$ for the safety application and size of the messages at 4096 bits [33]. The ad hoc coverage range and the data transfer rate are considered according to the IEEE $802.11 \mathrm{p}$ standard.

Vehicular density is considered constant, and total hops (i.e., nodes) competing for the channel vary between $5-40$, within five steps. In the case of heterogeneous illustration, our proposed MAMC-MAC protocol is correlated with standard protocol scenario, which displays appreciable output. The parameters used in the simulation are shown in Table 1.

Table 1. Parameters used in the simulation.

\begin{tabular}{cc}
\hline Specifications & Values \\
\hline Total nodes in our scheme & $5-40$ \\
Speed of node's & $10-40 \mathrm{~m} / \mathrm{s}$ \\
Area covered while simulation & $10,000 \times 10,000\left(\mathrm{~m}^{2}\right)$ \\
Data rate & $6000 \mathrm{kbps}$. \\
Time taken for simulation & $2.5 \mathrm{~min}$. \\
Total lanes in this scheme & Four \\
Scheme & Highway scenario \\
Transmission range & $0.3 \mathrm{~km}$ \\
Type of interface queue & DSRC/Queue \\
Length of interface queue & 50 \\
Network interface & WirelessPhyExt/Physical \\
Type of MAC interface used & $802.11 \mathrm{Ext}$ \\
Type of propagation framework & 2 ray ground \\
Size of message & 4096 Bits \\
Type of modulation used & Binary phase shift keying \\
Type of antenna & Omnidirectional antenna \\
\hline
\end{tabular}

\subsection{Performance Units}

For security message distribution, our proposed protocol performs well by considering the following units: packet delivery ratio, throughput, packet loss ratio, average end-to-end delay, probability of successful delivery, reliability, safety message travel time, and packet inter-reception time. 


\subsubsection{Packet Delivery Ratio (PDR)}

It is defined as the ratio of the number of successfully received packets to the total number of packets sent. PDR is analyzed as defined in Equation (3).

$$
\operatorname{PDR}=\frac{\sum_{i=1}^{n} y_{i}}{\eta^{T R F} \sum_{i=1}^{n} z_{i}}
$$

where ' $y_{i}$ ' represents the number of packets accepted by hop ' $i$ ', ' $z_{i}$ ' represents the number of packets diffused by hop ' $i$ ', and ' $\eta_{T R F}$ ' represents the average number of surrounding hops in the RF dissemination scale. The ' $\eta_{T R F}$ ' value is estimated by using vehicular density. As shown in Figure 6, the PDR of MAMC-MAC is compared with the IEEE 802.11 standard and various other protocols. PDR is maximized with the increment in the number of hops. This is because of the rise in the probability of packet delivery. The protocol performs best transferring message for four nodes.

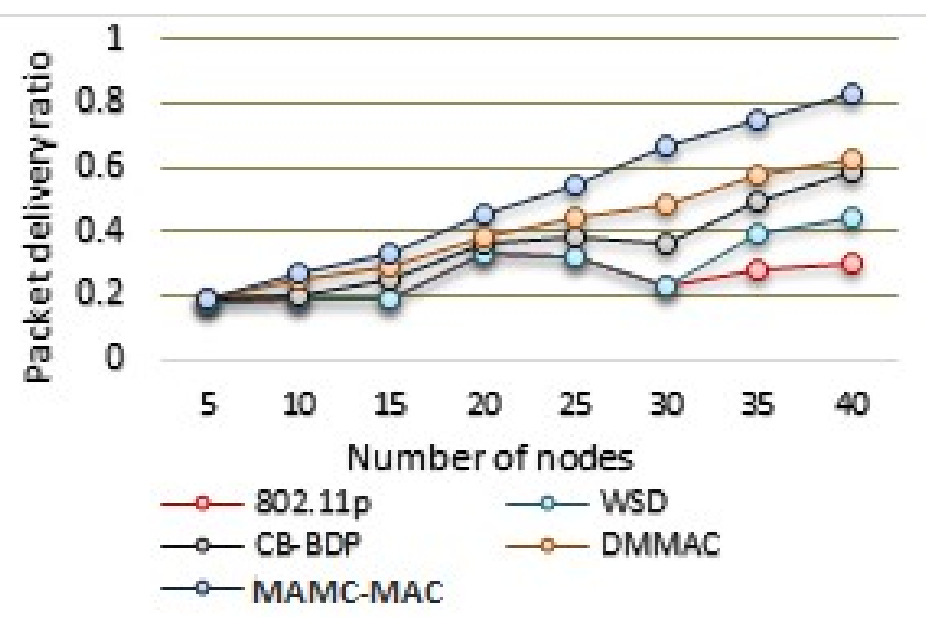

Figure 6. PDR comparison.

\subsubsection{Throughput}

It is determined as the amount of successful distribution of knowledge per unit period in a given network and systematically explained as defined in Equation (4).

$$
\text { Throughput }=\frac{\sum_{i=1}^{n} y_{i}}{T_{n}}
$$

From the Equation (4), ' $y_{i}$ ' represents the number of packets accepted by hops ' $i$ ', ${ }^{\prime} T_{n}$ ' represents the time taken for simulation in minutes, and ' $n$ ' represents the overall nodes within the network. A throughput comparison of our proposed scheme with other protocols is shown in Figure 7. The MAMC-MAC scheme performs well in throughput maximization when compared with other four protocols. Our proposed work shows an increase in throughput up to 35 nodes, whereas other protocols' throughput is increased up to 25 nodes only. This shows that the MAMC-MAC protocol has the maximum message delivery rate. 


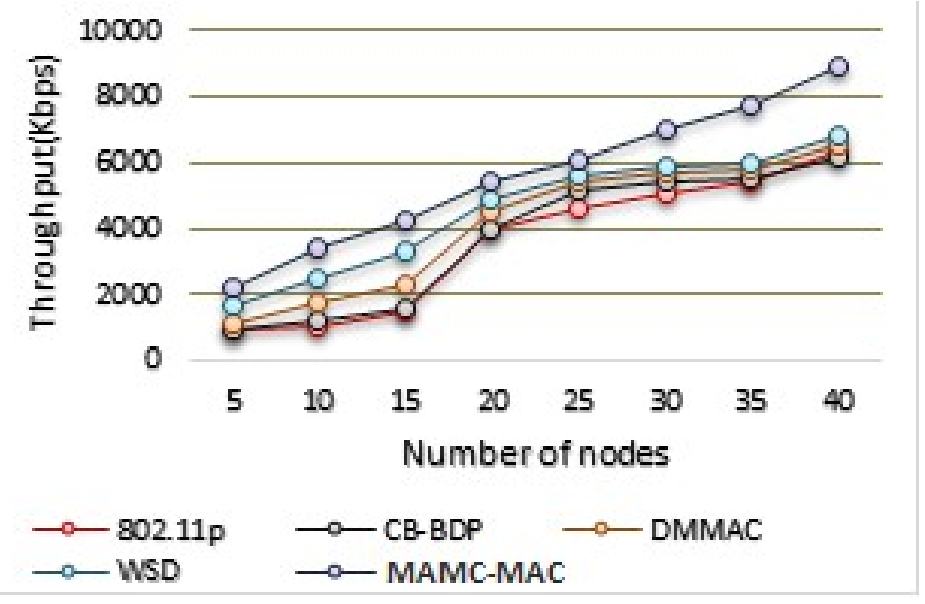

Figure 7. Throughput comparison.

\subsubsection{Packet Loss Ratio $\left(P_{L R}\right)$}

It is determined as the amount of data packets that fail to reach their target (i.e., amount of packet loss-APL) to the total number of packets transmitted (i.e., overall amount of packets disseminated-APD), as defined in Equation (5). The main reason for packet loss is due to network congestion.

$$
P_{L R}=\frac{A P L}{A P D}
$$

Figure 8 shows that MAMC-MAC performs well with a high possibility of message delivery.

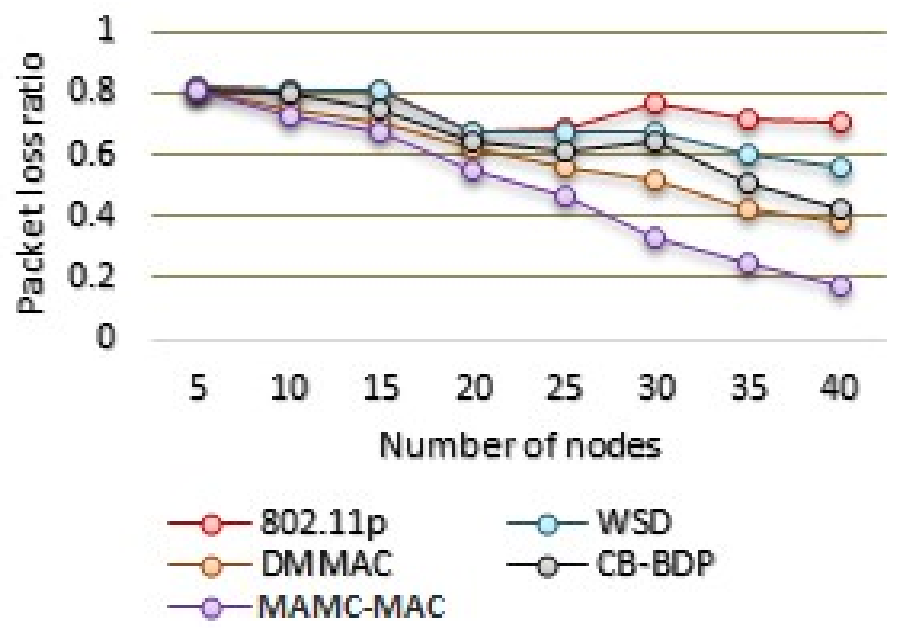

Figure 8. Packet loss ratio comparison.

\subsubsection{Average End-to-End Delay $\left(T_{\text {avg }}\right)$}

It is defined as the time taken in forwarding a packet from the starting hop to accepting the packet at the target hop. Figure 9 shows the comparison of the MAMC-MAC protocol with IEEE 802.11p and WSD. Here, the WSD scheme is compared for recognizing delivery delay as a stringent QoS requirement. It is observed that a delay arises due to an increase in the number of nodes. MAMC-MAC performs moderately better in terms of delay experienced. 


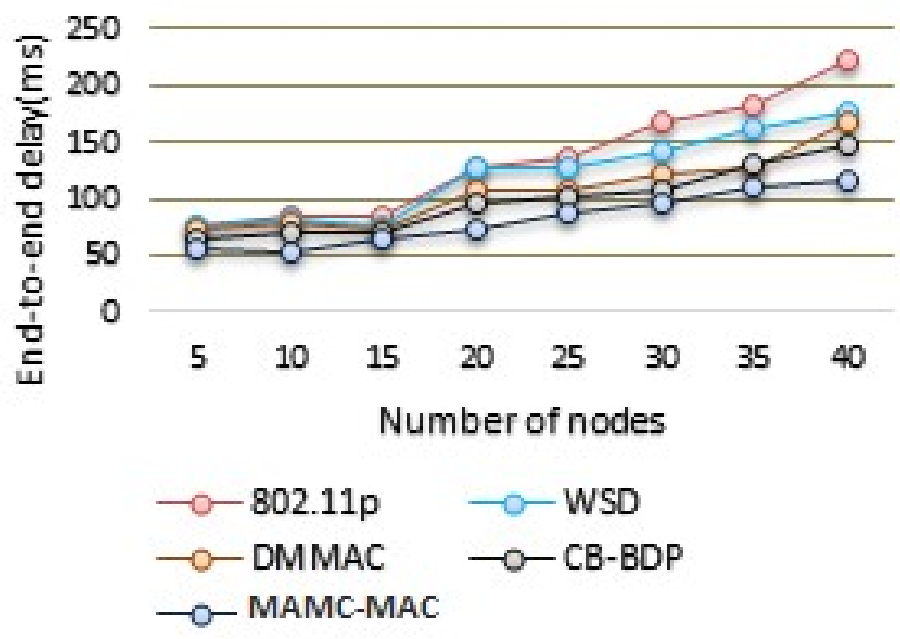

Figure 9. Average end-to-end delay comparison.

\subsubsection{Probability of Successful Delivery (PSD)}

High reliability is essential during safety message dissemination. PSD depends on the entire network capability as well as reliable data delivery. When compared with other protocols, MAMC-MAC displays increased possibility of effectiveness in delivering of messages. Figure 10 shows that the probability of successful delivery in standard protocols is minimized with maximization in vehicular density. However, the proposed MAMCMAC protocol has a possibility in the range from $75 \%$ to $90 \%$ for low density, until 20 nodes. As the density increases, the possibility decreases. This reveals that an increase in number of nodes decreases the number of message deliveries.

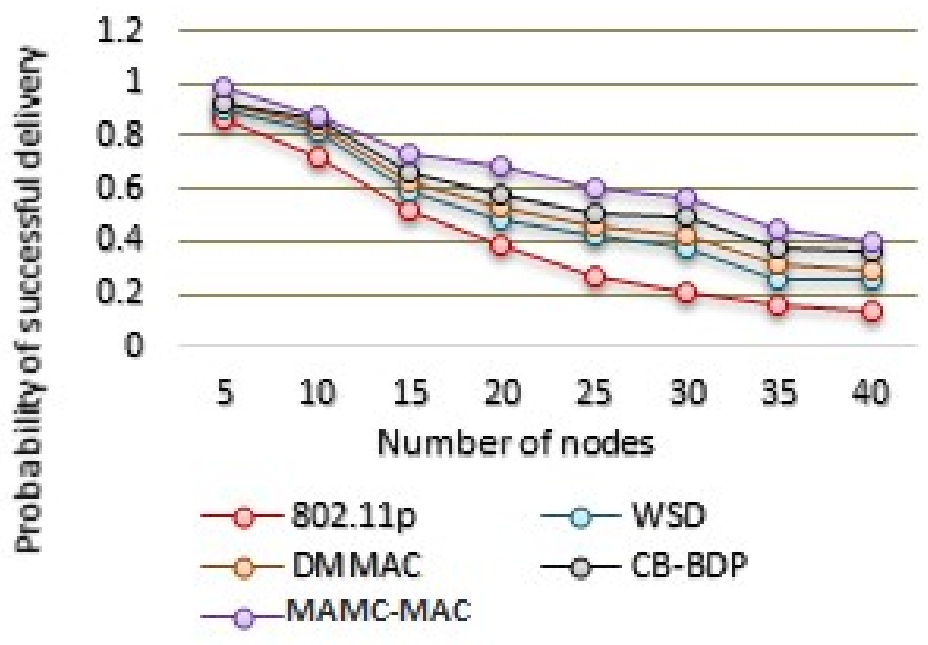

Figure 10. PSD comparison.

\subsubsection{Reliability}

It is defined as the probability of successful message transmission and reception within the cluster and its single-hop remote adjacent cluster. When we focus on safety message diffusion, reliability is one of the utmost key variables. A reliability comparison of our proposed work with other protocols is shown in Figure 11. Under a similar vehicular scenario, the DMMAC protocol only is focused on delivering safety messages. It is noticed that, under the specified simulation conditions, both MAMC-MAC and DMMAC accomplish uniformly good reliability. It is observed that the system accuracy is high in low-density area and marginally minimizes with the increase in vehicular scale. 


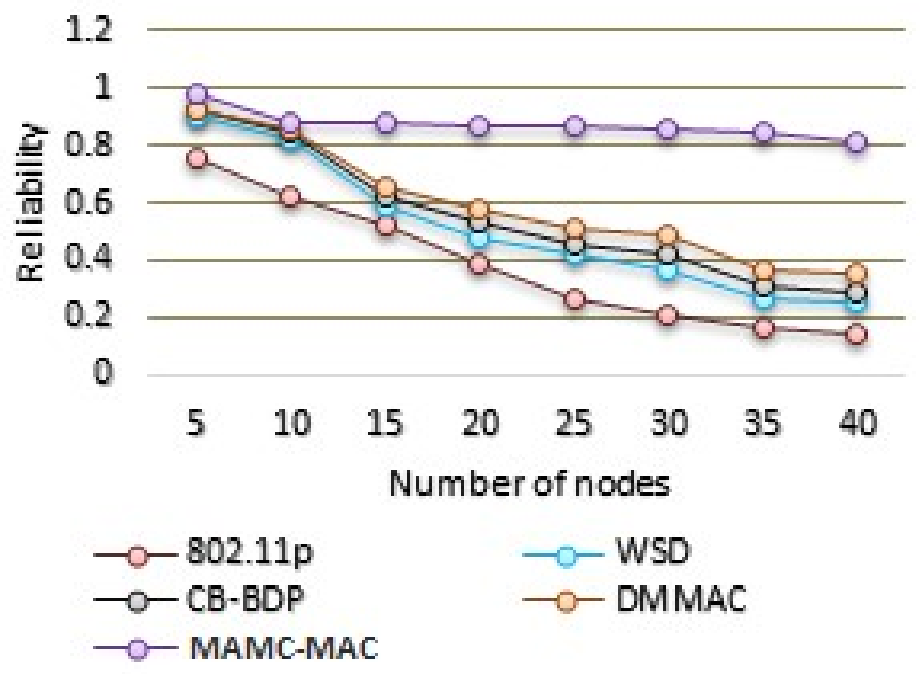

Figure 11. Reliability comparison.

\subsubsection{Safety Message Travel Time}

It is the time period taken by a safety message forwarded by a hop to reach its singlenode neighbor. Travel time decrease based on the number of nodes is shown in Figure 12. Besides this, as the hop scale is maximized, hopping is incremented, which results in faster message distribution. Furthermore, safety message travel time is increased with a decrease in the node density, which results in clashing of hops in finding a surrounding hop to transfer the message ahead. MAMC-MAC is perceived as having good accomplishments compared to the other protocols because of two limits. Primarily, cluster-head selection is not required in MAMC-MAC. This minimizes the extra periods that would have been taken in the process. Next, because the vehicular density is regarded as stable for the simulation extent, when the nodes are on the roads, HELLO message control is accomplished only once, which helps in minimizing the travel time.

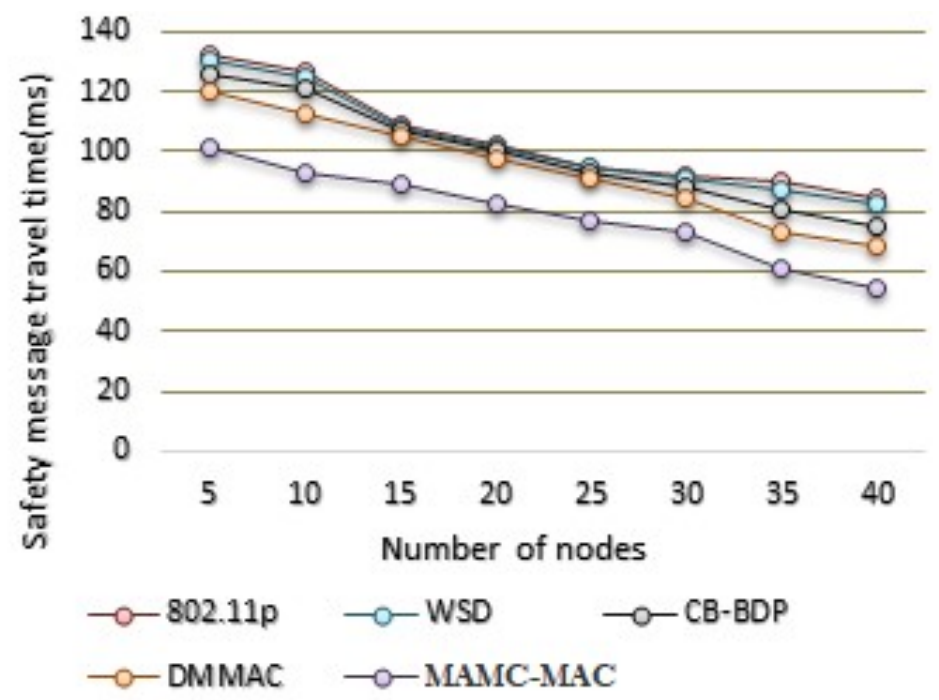

Figure 12. Message travel time comparison.

\subsubsection{Packet Inter-Reception Time (PIRT)}

From the results obtained and shown in Figure 13, it is noticed that PIRT increases for all of the protocols with increasing node density. Additionally, PIRT for MAMC-MAC is less when compared with other protocols. This improvement is because of the adoption of 
mobility-based clustering scenarios that the proposed protocol accomplishes well under dense vehicular environment.

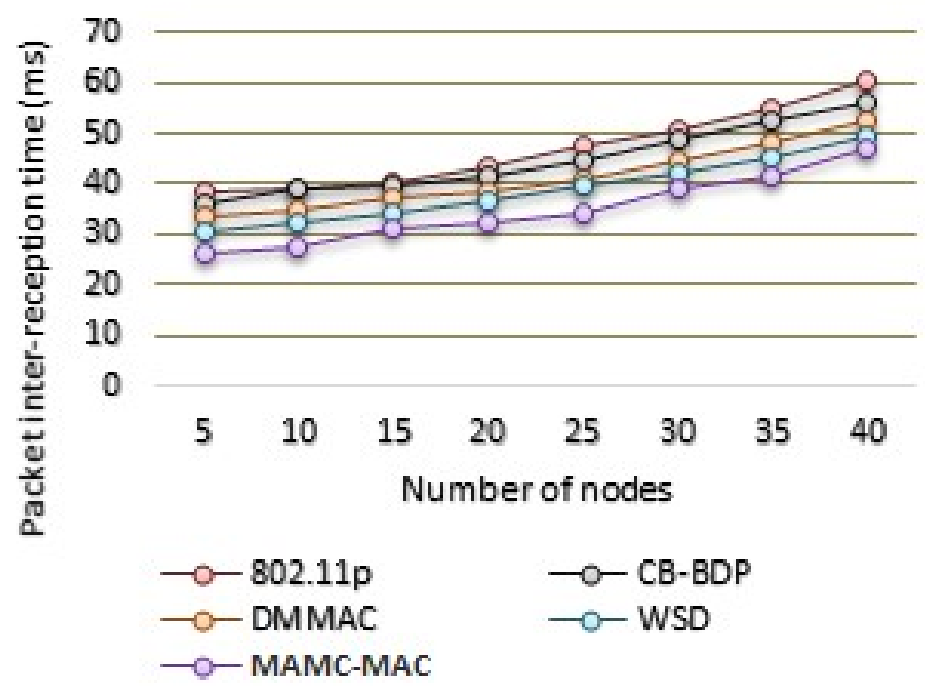

Figure 13. PIRT comparison.

\section{Conclusions}

In this work, we propose a unique mobility-based MAC protocol for traffic safety applications in VANET. With no cluster-head selection requirement, the communication among vehicles is performed by using a clustering-based TDMA schema for hopsector message distribution, which is completely in a distributed environment. Stability is increased by clustering the nodes on the basis of their speed. The cluster maintenance overhead is reduced by not electing the $\mathrm{CH}$. This scheme supports the reality that the highest priority must be given to the real-time traffic possessing higher sensitiveness to attain time slots compared to non-safety-based traffic, which is given a lower priority. Depending on its mobility characteristics, the TDMA mechanism assigns periods of time to the clusters. Reliability and timely delivery of safety messages are ensured by allowing frame synchronization between different clusters. The performance outcome shows that the network spends the minimum period of time compared with other scenarios. This is because of the small size of the cluster formation. Compared with other protocols, the MAMC-MAC protocol performs better delivering messages securely while maintaining high reliability even in a heavy traffic environment along with guaranteeing throughput.

The proposed MAMC-MAC protocol is efficient when scaling over a number of hops for delivering the messages in real-time traffic and results in increased bandwidth utilization and lowering interference. However, the proposed work is constrained to the traffic moving in a similar direction with uniform density. When the density is non-uniform, the interference is increased and bandwidth utilization is decreased; this is why we assume the vehicular density to be uniform.

In the future, we plan to extend our proposed work for various traffic scenarios depending on the assigned priority, facilitating non-safety messages, and incorporating adaptive contention window scheme during slot assignment. Additionally, the research shall be extended to designing protocols that consider dynamic single-hop and multi-hop dissemination and are capable of scheduling messages depending on their level of priority. Furthermore, a study shall be performed on the services that are applied irrespective of the location.

Author Contributions: Conceptualization, R.C. and N.G.; methodology, R.C. and N.G.; validation, R.C. and N.G.; writing-original draft preparation, R.C., N.G. and A.S.T.; writing-review and editing, R.C., N.G. and A.S.T. All authors have read and agreed to the published version of the manuscript. 
Institutional Review Board Statement: Not applicable.

Informed Consent Statement: Not applicable.

Conflicts of Interest: The authors declare no conflicts of interest.

\begin{tabular}{|c|c|}
\hline \multicolumn{2}{|c|}{ Abbreviations } \\
\hline \multicolumn{2}{|c|}{ The following abbreviations are used in this manuscript: } \\
\hline $\mathrm{CCHI}$ & Control Channel Interval \\
\hline $\mathrm{CHs}$ & Cluster Heads \\
\hline CMs & Cluster Members \\
\hline CSMA/CA & Carrier Sense Multiple Access with Collision Avoidance \\
\hline DSRC & Dedicated Short-Range Communication \\
\hline HTP & Hidden Terminal Problem \\
\hline MAC & Medium Access Control \\
\hline MAMC-MAC & Mobility-Aware Multi-hop Clustering-based MAC \\
\hline PDR & Packet Delivery Ratio \\
\hline PSD & Probability of Successful Delivery \\
\hline QoS & Quality of Service \\
\hline SAS & Slot-Assignment State \\
\hline SCHI & Service Channel Interval \\
\hline SINR & Signal-to-Interference-and-Noise Ratio \\
\hline TDMA & Time Division Multiple Access \\
\hline VANET & Vehicular Ad Hoc Network \\
\hline V2I & Vehicle-to-Infrastructure \\
\hline V2V & Vehicle-to-Vehicle \\
\hline WAVE & Wireless Access in Vehicular Environments \\
\hline
\end{tabular}

\section{References}

1. Rawashdeh, Z.Y.; Mahmud, S.M. Media access technique for cluster-based vehicular ad hoc networks. In Proceedings of the 2008 IEEE 68th Vehicular Technology Conference, Calgary, AB, Canada, 21-24 September 2008; IEEE: New York, NY, USA, 2008; pp. 1-5. [CrossRef]

2. Sheu, T.L.; Lin, Y.H. A Cluster-based TDMA System for Inter-Vehicle Communications. J. Inf. Sci. Eng. 2014, 30, $213-231$.

3. Song, C. Performance analysis of the IEEE 802.11 p multichannel MAC protocol in vehicular ad hoc networks. Sensors 2017, 17, 2890. [CrossRef] [PubMed]

4. Zhang, M.; Ali, G.M.N.; Chong, P.H.J.; Seet, B.C.; Kumar, A. A novel hybrid mac protocol for basic safety message broadcasting in vehicular networks. IEEE Trans. Intell. Transp. Syst. 2019, 21, 4269-4282. [CrossRef]

5. Alves Junior, J.; Wille, E.C. Routing in vehicular ad hoc networks: Main characteristics and tendencies. J. Comput. Netw. Commun. 2018, 2018. [CrossRef]

6. Rebei, A.; Hadded, M.; Touati, H.; Boukhalfa, F.; Muhlethaler, P. MAC-aware Routing Protocols for Vehicular Ad Hoc Networks: A Survey. In Proceedings of the 2020 International Conference on Software, Telecommunications and Computer Networks (SoftCOM), Split, Croatia, 17-19 September 2020; IEEE: New York, NY, USA, 2020; pp. 1-6. [CrossRef]

7. Gupta, N.; Manaswini, R.; Saikrishna, B.; Silva, F.; Teles, A. Authentication-based secure data dissemination protocol and framework for 5G-enabled VANET. Future Internet 2020, 12, 63. [CrossRef]

8. Gupta, N.; Prakash, A.; Tripathi, R. Mobility dependent clustering-based data transmission under variable data rate for different node densities in vehicular ad hoc network. Int. J. Adv. Intell. Paradig. 2017, 9, 246-262. [CrossRef]

9. Sumathi, J.; Velusamy, R.L. A review on distributed cluster based routing approaches in mobile wireless sensor networks. J. Ambient. Intell. Humaniz. Comput. 2020, 1-15. [CrossRef]

10. More, S.; Naik, U. Optimal Multipath routing for video transmission in VANETs. Wirel. Pers. Commun. 2021, 116, 805-827. [CrossRef]

11. Urmonov, O.; Kim, H. Multi-Hop Dynamic Map Data Propagation Algorithm for Clustered Vehicular Networks. Electronics 2020, 9, 1728. [CrossRef]

12. Almalag, M.S.; Olariu, S.; Weigle, M.C. Tdma cluster-based mac for vanets (tc-mac). In Proceedings of the 2012 IEEE International Symposium on a World of Wireless, Mobile and Multimedia Networks (WoWMoM), San Francisco, CA, USA, 25-28 June 2012; IEEE: New York, NY, USA, 2012; pp. 1-6. [CrossRef]

13. Ren, M.; Khoukhi, L.; Labiod, H.; Zhang, J.; Veque, V. A new mobility-based clustering algorithm for vehicular ad hoc networks (VANETs). In Proceedings of the NOMS 2016-2016 IEEE/IFIP Network Operations and Management Symposium, Istanbul, Turkey, 25-29 April 2016; IEEE: New York, NY, USA, 2016; pp. 1203-1208. [CrossRef] 
14. Hu, J.; Lyu, W.; Zhong, S.; Huang, J. Motion Prediction Based TDMA Protocol in VANETs. Electronics 2020, 9, 1792. [CrossRef]

15. Kim, H.S.; Paek, J.; Culler, D.E.; Bahk, S. PC-RPL: Joint control of routing topology and transmission power in real low-power and lossy networks. ACM Trans. Sens. Netw. (TOSN) 2020, 16, 1-32. [CrossRef]

16. Sharma, A.; Kumar, N. Third Eye: An Intelligent and Secure Route Planning Scheme for Critical Services Provisions in Internet of Vehicles Environment. IEEE Syst. J. 2021, 1-12. [CrossRef]

17. Rathee, G.; Sharma, A.; Iqbal, R.; Aloqaily, M.; Jaglan, N.; Kumar, R. A blockchain framework for securing connected and autonomous vehicles. Sensors 2019, 19, 3165. [CrossRef] [PubMed]

18. Sharma, A.; Kumar, R. An optimal routing scheme for critical healthcare HTH services-An IOT perspective. In Proceedings of the 2017 Fourth International Conference on Image Information Processing (ICIIP), Shimla, India, 21-23 December 2017; pp. 1-5. [CrossRef]

19. Poongodi, M.; Hamdi, M.; Sharma, A.; Ma, M.; Singh, P.K. DDoS Detection Mechanism Using Trust-Based Evaluation System in VANET. IEEE Access 2019, 7, 183532-183544. [CrossRef]

20. Deepthi, P.; Sivakumar, S.; Murugesan, R. Development of Multihop clustering algorithm for the simulation of VANET. Int. J. Comput. Appl. Math. 2017, 12. [CrossRef]

21. Zelikman, D.; Segal, M. Reducing interferences in VANETs. IEEE Trans. Intell. Transp. Syst. 2014, 16, 1582-1587. [CrossRef]

22. Luo, G.; Yuan, Q.; Zhou, H.; Cheng, N.; Liu, Z.; Yang, F.; Shen, X.S. Cooperative vehicular content distribution in edge computing assisted 5G-VANET. China Commun. 2018, 15, 1-17. [CrossRef]

23. Khan, Z.; Fan, P. A multi-hop moving zone (MMZ) clustering scheme based on cellular-V2X. China Commun. 2018, 15, 55-66. [CrossRef]

24. Najafzadeh, S.; Ithnin, N.; Abd Razak, S.; Karimi, R. BSM: Broadcasting of safety messages in vehicular ad hoc networks. Arab. J. Sci. Eng. 2014, 39, 777-782. [CrossRef]

25. Omar, H.A.; Zhuang, W.; Li, L. VeMAC: A TDMA-based MAC protocol for reliable broadcast in VANETs. IEEE Trans. Mob. Comput. 2012, 12, 1724-1736. [CrossRef]

26. Haas, J.J.; Hu, Y.C. Communication requirements for crash avoidance. In Proceedings of the Seventh ACM International Workshop on VehiculAr InterNETworking, Chicago, IL, USA, 24 September 2010; pp. 1-10. [CrossRef]

27. Lee, K.C.; Lee, U.; Gerla, M. Survey of routing protocols in vehicular ad hoc networks. In Advances in Vehicular Ad-Hoc Networks: Developments and Challenges; IGI Global: Hershey, PA, USA, 2010; pp. 149-170. [CrossRef]

28. Mu'azu, A.A.; Jung, L.T.; Lawal, I.A.; Shah, P.A. A QoS approach for cluster-based routing in VANETS using TDMA scheme. In Proceedings of the 2013 International Conference on ICT Convergence (ICTC), Jeju, Korea, 14-16 October 2013; IEEE: New York, NY, USA, 2013; pp. 212-217. [CrossRef]

29. Tian, D.; Wang, Y.; Xia, H.; Cai, F. Clustering multi-hop information dissemination method in vehicular ad hoc networks. IET Intell. Transp. Syst. 2013, 7, 464-472. [CrossRef]

30. Ghandour, A.J.; Di Felice, M.; Artail, H.; Bononi, L. Dissemination of safety messages in IEEE 802.11 p/WAVE vehicular network: Analytical study and protocol enhancements. Pervasive Mob. Comput. 2014, 11, 3-18. [CrossRef]

31. Hafeez, K.A.; Zhao, L.; Mark, J.W.; Shen, X.; Niu, Z. Distributed multichannel and mobility-aware cluster-based MAC protocol for vehicular ad hoc networks. IEEE Trans. Veh. Technol. 2013, 62, 3886-3902. [CrossRef]

32. Allouche, Y.; Segal, M. Cluster-based beaconing process for VANET. Veh. Commun. 2015, 2, 80-94. [CrossRef]

33. Chen, Q.; Jiang, D.; Taliwal, V.; Delgrossi, L. IEEE 802.11 based vehicular communication simulation design for NS-2. In Proceedings of the 3rd International Workshop on Vehicular Ad Hoc Networks, Los Angeles, CA, USA, 29 September 2006; pp. 50-56. [CrossRef] 